Annuaire suisse de politique de développement

25-2 | 2006

Paix et sécurité : les défis lancés à la coopération internationale

\title{
Promotion de la paix et coopération internationale : histoire, concept et pratique
}

Thania Paffenholz

\section{CpenEdition}

\section{Journals}

Édition électronique

URL : http://journals.openedition.org/aspd/242

DOI : $10.4000 /$ aspd. 242

ISSN : 1663-9669

Éditeur

Institut de hautes études internationales et du développement

Édition imprimée

Date de publication : 1 octobre 2006

Pagination : 19-45

ISBN : 2-88247-064-9

ISSN : $1660-5934$

Référence électronique

Thania Paffenholz, « Promotion de la paix et coopération internationale : histoire, concept et pratique », Annuaire suisse de politique de développement [En ligne], 25-2 | 2006, mis en ligne le 18 décembre 2009, consulté le 08 septembre 2020. URL : http://journals.openedition.org/aspd/242 DOI : https://doi.org/10.4000/aspd.242 


\title{
Promotion de la paix et coopération internationale: histoire, concept et pratique
}

\author{
Thania Paffenholz*
}

Introduction'

Dans le débat public sur les possibilités d'empêcher les conflits armés au sein d'Etats ou d'y mettre un terme, on ne perçoit généralement que les efforts de négociation des diplomates officiels ou des organisations internationales, avec leurs succès et leurs revers. Si la diplomatie a permis de conclure des traités de paix au Salvador ou au Soudan, elle a échoué jusqu'ici au Proche-Orient ou au Sri Lanka. La question se pose donc de savoir dans quelle mesure d'autres acteurs ou approches seraient en mesure de contribuer à court, à moyen et à long terme à faire baisser la violence, donc à promouvoir la paix. Autrement dit, la coopération au développement a-t-elle un rôle à jouer dans la promotion de la paix, ou les objectifs des mesures diplomatiques et de celles de la politique de développement sont-ils trop disparates?

Pour étudier cette question de plus près, il faut d'abord comprendre les rapports entre les conflits armés, la sécurité et la paix, d'une part, et la coopération au développement et l'aide humanitaire, de l'autre. Car si l'aide humanitaire a toujours été présente dans les guerres et les crises, la coopération au développement ne s'est vue confrontée aux conflits armés qu'à partir du début des années 1990. Depuis lors, le nombre de conflits armés dans les pays en développement a crû considérablement. Aujourd'hui, presque la moitié des pays partenaires de la coopération au développement connaissent des tensions politiques ou des conflits armés, ou sont alors frappés par les séquelles d'un conflit armé ou d'une guerre.

Quelques acteurs de la coopération au développement, comme de nombreuses organisations non gouvernementales (ONG) ecclésiastiques, étaient déjà actifs avant 1990 dans des pays en conflit armé, mais le travail dans les pays en guerre n'était pas encore l'objet de grands débats internationaux au sein de la coopération au développement.

L'origine de l'ample discussion concernant la coopération au développement dans les pays en conflit est la crise du Rwanda en 1994, où de nombreuses organisations de coopération au développement n'avaient pas vu venir le génocide. Après coup, les travaux de recherche montrèrent que l'aide au développement avait même eu involontairement des effets négatifs sur la dynamique

* Collaboratrice scientifique et de l'enseignement, Institut universitaire d'études du développement (IUED), Genève.

1 L'original de l'article est en anglais. La forme masculine a été utilisée partout, mais recouvre toujours le masculin et le féminin. 
conflictuelle, non seulement au Rwanda ${ }^{2}$, mais aussi dans d'autres pays ${ }^{3}$. Cette question se fait également plus pressante du fait que les acteurs de la coopération au développement se remettent aujourd'hui beaucoup plus vite au travail de reconstruction dans les pays d'après-guerre que par le passé.

Dans les pays en conflit, la coopération au développement est confrontée à toute une série de problèmes. Le déroulement et la dynamique des conflits armés évoluent rapidement. La question est donc de savoir si et quand la coopération au développement doit cesser ses activités pour les transférer à l'aide humanitaire (aide humanitaire) et, inversement, quand cette dernière doit se retirer et céder la place à la coopération au développement. Une autre question brûlante est de trouver des modes d'action différents, étant donné que les partenaires habituels de la coopération au développement ne peuvent souvent pas travailler dans toutes les parties du pays. La réalité du travail dans les pays en conflit met donc en question l'idée d'une continuité directe entre l'aide humanitaire et la coopération au développement: dans certaines parties du pays, la coopération au développement classique reste possible, mais non dans d'autres, qui sont plus touchées par les effets du conflit armé. De telles régions «paisibles» sont par exemple le sud de l'Ouganda, le nord du Soudan ou le sud du Sri Lanka. Dans la pratique, les acteurs de la coopération au développement restent la plupart du temps sur place là où cela est possible, tandis que l'aide humanitaire se concentre sur les régions en crise. Il y aussi à cela des raisons politiques: concéder qu'un pays entier est touché par un conflit armé équivaut à une déclaration politique, ce qu'aussi bien les acteurs du développement que leurs partenaires gouvernementaux cherchent à éviter.

Le but du présent article est de donner un aperçu critique des stratégies que les acteurs de la coopération au développement et de l'aide humanitaire peuvent appliquer pour promouvoir la paix, que ce soit seuls ou en liaison avec les initiatives diplomatiques. Il s'agit avant tout de remédier aux conséquences négatives des conflits armés, d'éviter les éventuels effets négatifs de la coopération au développement sur la dynamique conflictuelle et de contribuer à édifier des sociétés pacifiques.

L'article est conçu comme suit. La partie suivante étudie les notions fondamentales de «conflit» et de «promotion de la paix ». La troisième partie brosse l'histoire de la promotion de la paix, en se concentrant sur les liens entre celle-ci et le développement. La quatrième partie examine dans quelle mesure les conditions de la coopération au développement dans les pays en conflit se distinguent des conditions «normales». La cinquième partie présente des modèles de la manière dont les acteurs de la coopération au développement pourraient contribuer à promouvoir la paix aux trois niveaux de la politique du développement, des politiques sectorielles et des projets. La sixième partie expose des conclusions et lance de brefs défis. Le but est plus de donner un aperçu de la problématique générale que de fournir des analyses scientifiques détaillées sur chaque thème.

2 P. Uvin, Aiding Violence: The Development Enterprise in Rwanda, West Hartford, Connecticut, Kumarian Press, 1998.

3 M.B. Anderson, Do No Harm: How Aid Can Support Peace - or War, Boulder, Colorado, Lynne Rienner, 1999. 
Avant de pouvoir discuter stratégie, il convient d'élucider des notions importantes.

Le conflit est un différend d'intérêts, d'opinions ou de comportements entre individus ou groupes. Il s'agit donc d'un phénomène courant dans la vie sociale. Si un conflit est traité de façon constructive, il peut avoir des retombées extrêmement positives à la fois pour l'individu et l'ensemble de la société. Mais les conflits peuvent aussi déclencher la violence s'ils sont traités de façon destructive.

La littérature scientifique contient différentes définitions des conflits armés. Le Programme de données sur les conflits de l'Université d'Uppsala ${ }^{4}$ définit comme «conflit armé» les confrontations qui font au moins 25 victimes par an, et n'utilise le terme de «guerre» («conflit armé massif») qu'à partir de 1000 victimes. Dans la pratique, il est rarement question de «guerre», parce que le recours à ce terme équivaut à une déclaration de nature politique. "Conflit armé» est la tournure la plus employée, comme dans le présent article. Depuis la fin de la Seconde Guerre mondiale, plus de 200 conflits armés se sont déroulés dans plus de 140 régions. En 2004, le nombre de conflits armés se situait selon la définition retenue - entre $30^{5}$ et $42^{6}$.

Pourquoi les conflits sont résolus par la violence est une question ardue. L'opinion de la rue est que la guerre représente la face hideuse de l'humanité. Les études scientifiques renvoient à une série de causes politiques, sociales et économiques, où la lutte pour le pouvoir représente un facteur central. La littérature scientifique connaît cependant encore d'autres sources de conflit, comme la pénurie des ressources naturelles dans les sociétés nomades ou la discrimination à l'égard de certains groupes sociaux; en Afrique, les frontières artificielles et d'autres séquelles de l'époque coloniale sont la cause de nombreuses confrontations armées. L'important est de constater que seule la combinaison d'une série de causes et de facteurs d'escalade conduit à des formes violentes de conflit. Il est donc indispensable d'analyser à fond la constellation conflictuelle d'un pays donné.

Dans la théorie, on distingue trois phases au cours d'un conflit armé:

๑ la période précédant la violence;

$\checkmark$ le conflit armé;

๑ la période suivant le conflit armé.

$4<$ http://www.pcr.uu.se/research/UCDP $>$.

5 L. Harbom, P. Wallensteen, «Armed Conflict and Its International Dimensions: 1946-2004», Journal of Peace Research, vol. 42, nº 5, 2005, pp. 623-635.

6 W. Schreiber, Das Kriegsgeschehen 2003. Daten und Tendenzen der Kriege und bewaffneten Konflikte, Wiesbaden, VS Verlag für Sozialwissenschaften, 2004. 


\section{Graphique 1: Les trois phases d'un conflit armé}

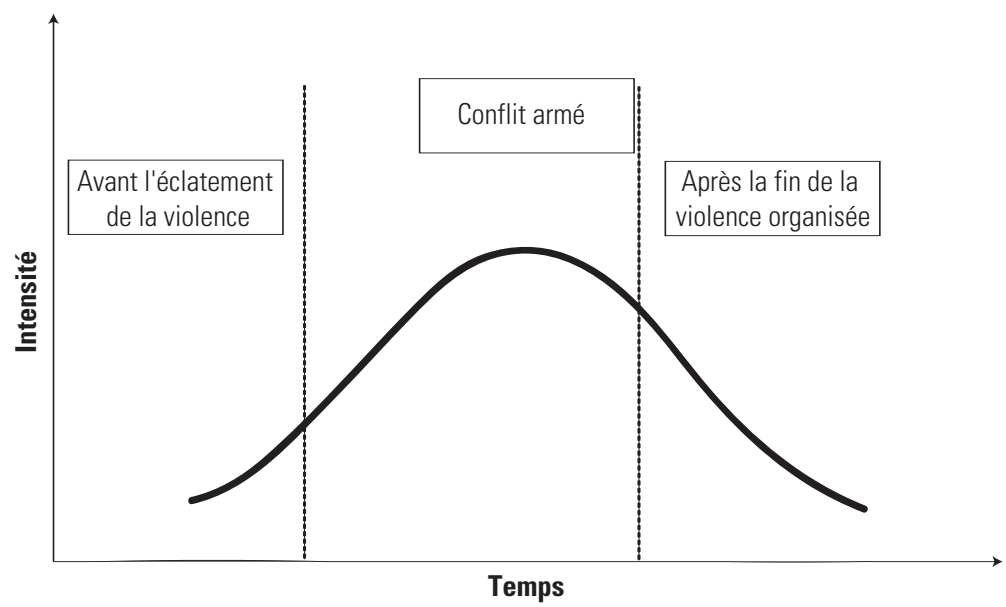

Dans la réalité, les conflits armés et les guerres ne suivent pas toujours cette courbe idéale d'intensité. Dans le conflit armé au Népal, par exemple, il y eut deux armistices et deux cycles de négociation pendant lesquels le niveau de violence baissa, bien que le conflit ne fût pas terminé; après l'échec des négociations, le conflit armé reprit et le niveau de la violence augmenta de nouveau nettement.

Il arrive aussi que les différentes phases d'un conflit se chevauchent, comme c'est le cas actuellement au Sri Lanka, où se déroule un conflit entre deux armées dans le nord et l'est de l'île alors que des attentats ciblés sèment le trouble dans la capitale et qu'ailleurs, dans le sud du pays, on pratique la coopération au développement traditionnelle.

La promotion de la paix est un terme général désignant un processus à long terme, qui comprend toutes les activités contribuant à éviter ou à surmonter la violence organisée et à maintenir la paix. Le but général de la promotion de la paix est d'empêcher les éruptions de violence dans les conflits ou de transformer durablement les conflits armés en des formes pacifiques et constructives de résolution des différends. Comme les conflits, la promotion de la paix connaît trois phases: $1^{\circ}$ la prévention, pour empêcher l'escalade de la violence; $2^{\circ}$ la gestion du conflit (ou la restauration de la paix), pour mettre un terme à la violence et aboutir à un traité de paix; $3^{\circ}$ la consolidation de la paix, pour stabiliser celle-ci après la guerre. Cette dernière phase est souvent subdivisée en différentes étapes, car les cinq premières années après la cessation des hostilités présentent un risque élevé $(44 \%)$ de reprise de la violence. Après un délai de cinq ans, ce risque décline cependant considérablement et, après dix ans, il connaît une nouvelle chute ${ }^{7}$.

La définition présentée de la promotion de la paix n'en décrit pas encore suffisamment la portée ni la durée, entre autres parce qu'elle ne précise pas explici-

7 P. Collier et al., Breaking the Conflict Trap: Civil War and Development Policy, New York, Oxford University Press, 2003. 
tement quand la phase de promotion de la paix prend fin. Dès la fin des années 1960, le célèbre spécialiste de la paix Johan Galtung ${ }^{8}$ distinguait déjà entre paix «négative» (fin de la violence) et paix «positive» (pacification de la société à tous les niveaux), ce qui sous-entendait implicitement une interprétation large de la promotion de la paix, puisqu'elle englobait la paix positive (utopique). Le chercheur allemand Ernst-Otto Czempiel $^{9}$ définissait la paix comme processus et mettait donc aussi l'accent sur sa longueur. John Paul Lederach, spécialiste américain de la paix et médiateur, se range à cette interprétation, mais fixe tout de même au processus de promotion de la paix un délai concret de trente ans ${ }^{10}$.

On trouve une définition plus stricte de la promotion de la paix dans l'Agenda pour la paix de l'ONU ${ }^{11}$, où celle-ci vise à empêcher l'éruption ou la reprise de la violence, et se limite donc à la paix «négative» (fin des hostilités). Toutes les actions dans ce sens sont donc des mesures de promotion de la paix. Le délai peut être fixé entre un et trois ans (cinq, au maximum) après la fin d'un conflit armé. Dans la pratique internationale, l'interprétation prédominante aujourd'hui est que la paix est atteinte $a$ ) lorsqu'un pays offre suffisamment de sécurité à la population pour que les troupes internationales de défense de la paix puissent quitter le pays et $b$ ) que des structures démocratiques fiables ont été établies. Cette seconde condition présuppose qu'un gouvernement national opérationnel ait été constitué et légitimé par des élections valides sous surveillance internationale.

Etant donné ces différences de définition, le présent article adopte un compromis entre la notion stricte de l'Agenda pour la paix et l'interprétation large de la paix «positive». Autrement dit, le but de la promotion de la paix est d'empêcher les conflits armés ou d'y mettre fin, ainsi que d'en stabiliser la résolution pacifique après la fin des violences. Cette définition englobe toutes les mesures liées directement à ce but, dans un délai de cinq ans au moins et de dix au plus. La promotion de la paix a donc aussi pour tâche de créer les conditions favorables à la reconstruction continue, au développement et à la démocratisation, mais sans comprendre ces activités elles-mêmes.

Brève histoire de la promotion de la paix et tendances actuelles

Début de la promotion moderne de la paix et des recherches sur la paix

Dans l'histoire, la paix a toujours été promue par différents acteurs, mais ce n'est qu'au $\mathrm{XX}^{\mathrm{e}}$ siècle que la promotion de la paix a été institutionnalisée dans le droit international comme moyen de résolution pacifique des conflits entre Etats. Ce processus débuta en 1898 à la Conférence de la paix de La Haye et trouva son prolongement dans la fondation de la Société des Nations, puis, après

8 J. Galtung, «Violence, Peace, and Peace Research», Journal of Peace Research, vol. 6, n 3, 1969, pp. 167-191.

9 E. Czempiel, Schwerpunkte und Ziele der Friedensforschung, München, Kaiser, 1972.

10 J.P. Lederach, Building Peace: Sustainable Reconciliation in Divided Societies, Washington, DC, United States Institute of Peace Press, 1997.

11 B. Boutros-Ghali, Agenda pour la paix, 1992, accessible depuis <http://www.un.org/Depts/dhl/dhlf/ landmark_sc/topical.htm>. 
la Seconde Guerre mondiale, des Nations unies (ONU). La mission première de l'ONU est de maintenir la paix dans le monde ${ }^{12}$.

En tant que branche interdisciplinaire normative, les recherches sur la paix se sont constituées au cours des années 1960 dans le monde universitaire anglophone. Pendant la guerre froide, elles se concentraient surtout sur l'évitement d'une guerre atomique ou classique entre les deux camps adverses. Elles étaient donc fortement liées aux mouvements pacifistes. Une autre branche des recherches sur la paix est étroitement liée à la théorie des relations internationales et s'est intéressée très tôt à la cessation des conflits armés. Cette école, dite de la «gestion des conflits», entend mettre fin aux guerres en recourant à divers procédés et instruments diplomatiques. Elle est étroitement liée à l'institutionnalisation de la promotion de la paix dans le droit international public et considère que cette mission incombe surtout aux diplomates des organisations gouvernementales bi- ou multilatérales ${ }^{13}$. Sa méthodologie peut être qualifiée de fonctionnelle (outcome-oriented). Le but est d'identifier et de réunir les chefs représentatifs des parties au conflit pour négocier un armistice et/ou un traité de paix. On en connaît plusieurs exemples pratiques, comme les accords de Camp David ou le récent traité de paix au Soudan.

L'engagement d'acteurs de la société civile - surtout dans les conflits internationaux - a été considéré longtemps comme une entrave à l'action des diplomates professionnels ${ }^{14}$. Les acteurs non étatiques actifs dans la promotion de la paix, comme les quakers, étaient alors l'exception ${ }^{15}$. L'école scientifique qui les inspire est celle dite «de résolution des conflits»: il s'agit d'éliminer les sources de conflit et de rétablir les bonnes relations entre les parties, au niveau non seulement des leaders politiques et des élites, mais bien de la société tout entière. Dans les années 1970, cette école de pensée a investi la recherche en tentant de transposer aux conflits armés les stratégies de la résolution des conflits individuels. La reconstruction des relations à tous les niveaux de la société fait l'objet de différentes activités: ateliers de résolution des conflits, projets de dialogue pour promouvoir les échanges entre groupes et communautés, entraînement à la résolution des conflits pour développer les capacités d'acteurs susceptibles de provoquer le changement, comme les femmes, les jeunes, les journalistes et les réseaux des organisations pacifistes, etc. ${ }^{16}$

12 T. Paffenholz, «Ansätze ziviler Konfliktbearbeitung», in Zivile Konfliktbearbeitung. Eine internationale Herausforderung, Schriftenreihe des Österreichischen Studienzentrums - Studien für Europäische Friedenspolitik, Band 8, Münster, agenda Verlag, 2001, pp. 15-26.

13 T. Paffenholz, Konflikttransformation durch Vermittlung. Theoretische und praktische Erkenntnisse aus dem Friedensprozess in Mosambik (1995-1996). Mainz, Grunewald, 1998. Idem, «Ansätze ziviler Konfliktbearbeitung», op. cit. Idem, «Western Approaches to Mediation», in Peacebuilding : A Field Guide, L. Reychler, T, Paffenholz, (eds.), Boulder, Colorado, Lynne Rienner, 2001, pp. 75-81.

14 M.R. Berman, J.E. Johnson, Unofficial Diplomats, New York, Columbia University Press, 1977.

15 A. Curle, Making Peace, London, Tavistock Publications, 1971.

16 C. Mitchell, «Conflict, Social Change and Conflict Resolution: An Enquiry», in Berghof Handbook for Conflict Transformation, D. Bloomfield, M. Fischer, B. Schmelzle, Berlin, Berghof Research Center for Constructive Conflict Management, 2005, <http://www.berghof-handbook.net>. 
Importance croissante de la promotion de la paix depuis la fin de la guerre froide et l'Agenda pour la paix de l'onu (1992)

Ce n'est qu'avec la fin de la guerre froide, au début des années 1990, que la promotion de la paix s'est mise à gagner en importance sur le plan mondial. Depuis, sa cible s'est aussi déplacée; elle vise désormais moins à résoudre les conflits entre Etats qu'à gérer les conflits armés au sein d'un même Etat ${ }^{17}$. C'est qu'à cette époque-là, les conflits armés internes composaient déjà $80 \%$ à $90 \%$ de tous les conflits armés ${ }^{18}$, et la tendance est à la hausse.

A partir du milieu des années 1990, le nombre des activités de promotion de la paix crût de façon vertigineuse, presque parallèlement au débat international concernant l'adaptation des instruments disponibles aux nouveaux problèmes posés par les conflits armés internes. Le début de ce processus toujours en cours fut la publication, en 1992, du rapport du secrétaire général de l'onU, Boutros Boutros-Ghali, intitulé Agenda pour la paix. Ce document important posait le cadre de la gestion des conflits internationaux. On introduisit alors non seulement la nouvelle notion de promotion de la paix (peace-building), mais celle de consolidation de la paix après la guerre (post-conflict peace-building) passa également sur le devant de la scène. C'était une réaction au nouveau rôle de l'oNU et de la communauté internationale dans la reconstruction des sociétés frappées par la guerre.

Brève euphorie au début des années 1990, après la fin de nombreux conflits armés

Au début des années 1990, toute une série de conflits armés (Namibie, Angola, Mozambique, Cambodge ou Salvador) purent être résolus. La possibilité d'un monde meilleur semblait à portée de main, comme si ces conflits n'avaient été que des sous-produits de substitution de la guerre froide. On discutait déjà dans divers pays - dont la Suisse, notamment - de la manière dont on allait transférer les fonds affectés à l'armée à la coopération au développement et à la promotion de la paix. Pourtant, les guerres en Somalie et dans l'ex-Yougoslavie ainsi que le génocide au Rwanda mirent rapidement fin à cette euphorie.

Nouveaux débats après la crise rwandaise (1994): empêcher les conflits armés - le débat sur l'alerte précoce

Le génocide rwandais de 1994 suscita un débat sur la manière dont on pourrait éviter désormais un second Rwanda. C'est alors qu'on se mit à parler des possibilités de l'alerte précoce ${ }^{19}$. Les personnes impliquées postulaient qu'on disposerait très vite de méthodes quantitatives pour prédire la violence politique, ce qui permettrait d'intervenir politiquement à temps - espoir qui ne s'est malheu-

17 H. Miall, O. Ramsbotham, T. Woodhouse, Contemporary Conflict Resolution, Cambridge, Polity Press, 1999. M. Eriksson, P. Wallensteen, M. Sollenberg, «Armed Conflict, 1989-2002», Journal of Peace Research, vol. 40, n' 5, 2003, pp. 593-607.

18 M. Eriksson et al., op. cit., p. 594.

19 K. Rupesinghe, M. Kuroda, Early Warning and Conflict Resolution, Houndmills, Palgrave Macmillan, 1992. 
reusement pas réalisé jusqu'ici. Il est apparu au contraire que les systèmes quantitatifs d'alerte précoce ne peuvent prédire à eux seuls une éruption de violence politique. Le problème principal n'était - et n'est - d'ailleurs pas l'absence d'informations, mais le manque de volonté politique d'intervenir à temps. Le débat sur l'alerte précoce s'enlisa donc et se fondit dans la discussion plus générale sur la prévention des conflits, qui atteignit son sommet dans le rapport du secrétaire général de l'ONU Prévention des conflits armés ${ }^{20}$, où celle-ci est reconnue comme l'une des tâches fondamentales de la communauté des Etats.

De nos jours, on applique toute une série de systèmes quantitatifs et qualitatifs d'alerte précoce. Ainsi, l'International Crisis Group ${ }^{21}$ rédige régulièrement des analyses qualitatives des conflits; le projet d'alerte précoce FAST de l'ONG suisse Swisspeace exploite un système quantitatif qui fournit aux organisations de coopération au développement des informations sur la dynamique conflictuelle dans différents pays ${ }^{22}$.

\section{Coopération au développement et conflits armés}

La crise rwandaise accentua cependant aussi la discussion sur le rôle de la coopération au développement dans les pays en conflit, surtout quant à ses incidences négatives (involontaires) sur la dynamique conflictuelle ${ }^{23}$, qui se marquent dans quatre domaines.

$1^{\circ}$ Les modalités du transfert des ressources peuvent favoriser certains groupes ou régions et renforcer ainsi les inégalités existantes; après le tsunami de 2005, par exemple, la partie sud du Sri Lanka, soit celle tenue par le gouvernement, a reçu beaucoup plus d'aide que le nord et l'est du pays, tenus par les Liberation Tigers of Tamil Eelam (LTTE), ce qui a aggravé les tensions et fait monter la violence.

$2^{\circ} \mathrm{La}$ coopération au développement peut libérer des fonds en faveur de la guerre. Cet effet de remplacement a permis par exemple au gouvernement éthiopien de disposer de ressources militaires suffisantes pendant la guerre contre l'Erythrée du fait que les organisations internationales d'entraide se souciaient de la population nécessiteuse.

$3^{\circ}$ La coopération au développement donne parfois des signaux moraux ambigus. En cas de situation critique, par exemple, les coopérants étrangers sont souvent les seuls à être évacués, alors que les collaborateurs locaux et la population doivent rester sur place. L'impression donnée est que la valeur de la vie varie selon l'appartenance à tel ou tel groupe ${ }^{24}$.

$4^{\circ}$ Les projets de coopération au développement peuvent aggraver involontairement des conflits existants. Les activités de la coopération ont souvent pour but avoué de promouvoir les mutations sociales, par exemple en renforçant le rôle de la femme dans les sociétés traditionalistes ou en informant les exploi-

$21<$ http. 'Www.icg.org >.

$22<$ http://www.swisspeace.org/fast>.

23 P. Uvin, Aiding Violence, op. cit. M.B. Anderson, Do No Harm, op. cit.

24 M.B. Anderson, Do No Harm, op. cit. 
tants locaux du sol de leurs droits. De tels projets déclenchent naturellement des conflits, qui peuvent souvent déboucher sur une escalade de la violence. C'est pourquoi les acteurs de la coopération au développement doivent instamment se poser la question de savoir comment empêcher cette escalade. Les premières réflexions entreprises au milieu des années 1990 allaient dans trois directions :

\section{$1^{\circ}$ Approfondir l'analyse}

Il s'agit de mieux comprendre les causes et le potentiel d'escalade des conflits armés pour déterminer où et comment la coopération au développement peut intervenir préventivement. A cet effet, on a souvent eu recours à des analyses scientifiques qualitatives, à partir desquelles ont été mis au point toute une série d'instruments de travail (tools) conviviaux ${ }^{25}$. Un débat annexe étudie l'aspect économique des conflits armés (war economy), car dans les pays riches en ressources naturelles ou autres, les parties au conflit ont plus de facilité pour financer leurs guerres, par exemple en se livrant au trafic de diamants (Sierra Leone, Angola, Congo) ou de drogue (Colombie, Afghanistan); certains acteurs voient leur puissance économique s'accroître du fait de la guerre et n'entendent pas perdre ce nouveau pouvoir ${ }^{26}$.

Il en résulte qu'il devient difficile de distinguer les intérêts d'ordre politique et ceux de nature purement économique. Dans de tels cas, même la gestion diplomatique classique des conflits, qui se fonde sur la recherche d'un consensus politique, est remise en question ${ }^{27}$.

Un débat tout différent, mais sur le même sujet, s'est ouvert après le 11 septembre 2001 et l'intervention américaine en Irak: dans quelle mesure les guerres comme celle en Irak sont-elles conduites par des Etats occidentaux pour assurer leur approvisionnement en matières premières ? ${ }^{28}$

\section{$2^{\circ}$ Etudier les séquelles des conflits armés}

Ce centre d'intérêt a mis à l'ordre du jour des questions de politique de la sécurité comme la démobilisation et la réinsertion sociale des anciens combattants ou le problème des mines antipersonnel. Le débat a été repris désormais au niveau de la sécurité des personnes, c'est-à-dire d'une interprétation de la sécurité qui se concentre sur la protection des individus et des communautés plutôt que sur la sécurité d'Etat. Le concept en a été formulé pour la première fois par le Programme des Nations unies pour le développement (PNUD), dans son Rapport mondial sur le développement humain 1994.

\section{$3^{\circ}$ Promouvoir la paix positivement}

Au début, les acteurs de la coopération au développement faisaient valoir qu'à long terme, la plupart des activités de lutte contre la pauvreté contribueraient en elles-mêmes à promouvoir la paix, parce qu'ils voyaient dans la pauvreté la cause de très nombreux conflits. Vers la fin des années 1990, cependant, il

25 Cf. par exemple S. Fisher et al., Working with Conflict: Skills and Strategies for Action, Zed Book, London, 2000.

26 Voir la contribution de Gabi Hesselbein au présent ouvrage.

27 M. Berdal, D. Malone (eds.), Greed and Grievance : Economic Agendas of Civil Wars, Boulder, Colorado, Lynne Rienner, 2000.

28 Voir la contribution de Claude Serfati au présent ouvrage. 
devint toujours plus évident que les mesures de lutte contre la pauvreté n'instaureraient pas automatiquement et à elles seules les conditions favorisant une société pacifique, étant donné que les conflits ont des causes plus complexes.

\section{Augmentation fulgurante des activités}

de promotion de la paix à partir du milieu des années 1990

Jusqu'au milieu des années 1990 et contrairement à la coopération au développement, la promotion de la paix se concentrait essentiellement sur le rôle des acteurs externes dans la résolution des conflits internationaux. Des discussions menées au niveau scientifique et pratique, il ressortit que seul l'engagement d'acteurs différents (des diplomates aux acteurs de la société civile), travaillant avec une multitude de méthodes, conduirait au succès ${ }^{29}$. L'approche scientifique sous-jacente, soit celle de l'école dite «complémentaire», cherche à unir les logiques des deux écoles de la «gestion des conflits» et de la «résolution des conflits» en engageant à titre complémentaire les bons acteurs et les bonnes méthodes au bon moment. On dispose ici de trois systèmes: le contingency model de Fisher et Keashly ${ }^{30}$, le travail de Bercovitch et Rubin ${ }^{31}$ et, enfin, le multi-track diplomacy de Diamond et McDonald ${ }^{32}$. La nouvelle école n'a pas été critiquée très largement, mais n'a pas non plus suscité de débat fondamental au sein de la communauté des chercheurs. La raison en est probablement l'apparition de l'école de «transformation des conflits», qui a repris les conclusions de l'école «complémentaire».

L'idée qui inspire l'école «transformiste» est celle d'un processus transformant les conflits armés en conflits pacifiques. Le premier système transformiste complet a été élaboré par John Paul Lederach ${ }^{33}$, qui essaie de résoudre le dilemme entre gestion à court terme du conflit et mise sur pied de relations à long terme (élimination des sources de conflit). Il propose donc d'édifier à long terme des infrastructures de promotion de la paix qui soutiennent le potentiel de réconciliation existant au sein de la société. Les interventions de personnes extérieures doivent se borner à soutenir les acteurs internes du pays en conflit et à coordonner les efforts extérieurs en faveur de la paix. Pour réussir, cette méthode exige une profonde compréhension de la culture du pays en guerre ainsi qu'une longue patience. Un facteur clé du système de Lederach est l'approche «par le milieu» (middle-out): il s'agit d'identifier des personnalités ou groupes représentatifs des cadres moyens de la société (couche 2), puis de les aider à édifier

29 Pour l'état de la question, voir les trois ouvrages collectifs suivants: L. Reychler, T. Paffenholz, Construire la paix sur le terrain. Mode d'emploi, Bruxelles, Complexe, 2000. C.A. Crocker, F.O. Hampson, P. Aall, Turbulent Peace: The Challenges of Managing International Conflict, Washington, DC, United States Institute of Peace Press, 2001. A. Austin, M. Fischer, N. Ropers (eds.), Transforming Ethnopolitical Conflict, Wiesbaden, The Berghof Handbook, VS Verlag für Sozialwissenschaften, 2004.

30 R. Fisher, L. Keashly, «The Potential Complementarity of Mediation and Consultation with a Contingency Model of Third Party Intervention », Journal of Peace Research, vol. 28, n 1, 1991, pp. 29-42.

31 J. Bercovitch, J.Z. Rubin, Mediation in International Relations: Multiple Approaches to Conflict Management, London, St. Martin's Press, 1992.

32 L. Diamond, J. McDonald, Multi-Track Diplomacy: A Systems Approach to Peace, West Hartford, CT, Kumarian Press, 1996.

33 J.P. Lederach, Building Peace: Sustainable Reconciliation in Divided Societies, Washington, DC, United States Institute of Peace Press, 1997. 
la paix dans leur propre pays. On postule ici que ce travail se répercutera aussi bien au niveau supérieur (couche 1) que sur la base de la société (grass root level, couche 3 ). Cette méthode a beaucoup d'affinité avec le système de l'autonomisation (empowerment) pratiqué dans la coopération au développement.

Après l'échec des tentatives diplomatiques et militaires de paix en Somalie et le génocide au Rwanda, les idées de Lederach tombaient sur un sol fécond. Les activités internationales, nationales et locales de promotion de la paix se multiplièrent presque d'un bond. Alors que le travail en faveur de la paix était autrefois une activité bénévole par excellence, un nombre considérable d'acteurs professionnels non étatiques (ONG, groupements religieux, milieux économiques, groupes de citoyens, individus) s'engagent aujourd'hui dans des projets très divers de promotion de la paix ${ }^{34}$.

\section{Leçons et capitalisation des expériences}

Ces dix dernières années, malgré des activités accrues de promotion de la paix, le nombre de conflits armés n'a pas diminué substantiellement. Depuis le début du nouveau millénaire, les gens de terrain et les chercheurs s'efforcent donc d'étudier une décennie d'interventions dans les pays en conflit. Ils s'intéressent particulièrement à la question des effets des interventions pacificatrices sur le processus de paix. L'étude scientifique des expériences faites fournit des réponses à deux questions.

\section{$1^{\circ}$ Conditions de succès pour les traités de paix}

Les conditions essentielles pour un traité de paix durable sont la volonté de compromis des parties adverses ${ }^{35}$, l'identification du moment propice à un tel traité $^{36}$, l'implication des «durs » qui saboteraient sinon les négociations ${ }^{37}$, des accords de partage du pouvoir entre les groupes antagonistes ${ }^{38}$, la recherche d'un équilibre des forces dans la région, l'existence de divers canaux de médiation et de soutien ${ }^{39}$ et, enfin, la qualité même du traité de paix ${ }^{40}$.

34 Voir: European Centre for Conflict Prevention, People Building Peace: 35 Inspiring Stories from around the World, Utrecht, ECCP, 1999. P. Aall, «What Do NGOs Bring to Peacemaking?», in Turbulent Peace, C.A. Crocker, F.O. Hampson, P. Aall, op. cit., pp. 365-383. C. Orjuela, Civil Society in Civil War: Peace Work and Identity Politics in Sri Lanka, PhD Dissertation, Department of Peace and Development Research, Göteborg University, 2004. P. van Tongeren, M. Brenk, M. Hellema, J. Verhoeven, People Building Peace II: Successful Stories of Civil Society, Boulder, Colorado, Lynne Rienner, 2005.

35 B. Walter, «The Critical Barrier to Civil War Settlement», International Organization, vol. 51, $\mathrm{n}^{\circ} 3$, 1997, pp. 335-364.

36 I. Zartman, Ripe for Resolution: Conflict and Intervention in Africa, New York, Oxford University Press, 1989.

37 S.J. Stedman, «Spoiler Problems in Peace Processes», International Security, vol. 22, n 2, 1997, pp. 5-53.

38 W. Linder, Swiss Democracy: Possible Solutions to Conflict in Multicultural Societies, London, Macmillan, 1994.

39 T. Paffenholz, «Ansätze ziviler Konfliktbearbeitung», op. cit. M. Fitzduff, Beyond Violence: Conflict Resolution Processes in Northern Ireland, New York, Brookings Institute; United Nations University Press, 2002.

40 F.O. Hampson, Nurturing Peace: Why Peace Settlements Succeed or Fail, Washington, DC, United States Institute of Peace Press, 1996. 


\section{$2^{\circ}$ Expériences en matière de consolidation de la paix}

L'Agenda pour la paix de l'ONU a mis pour la première fois en exergue l'importance de la consolidation de la paix après la guerre. La question primordiale est de savoir comment stabiliser la paix pendant la période qui suit immédiatement la guerre. On trouvera ici deux démarches scientifiques différentes.

L'une se concentre sur les conditions requises pour qu'un traité de paix soit appliqué avec succès, soit le partage du pouvoir entre les anciennes parties au conflit, le soutien international apporté au processus, les garanties de sécurité offertes ainsi que les mécanismes de résolution des problèmes non résolus jusque-là ${ }^{41}$. Dans ce contexte, la notion de sécurité des personnes a encore gagné en importance ${ }^{42}$.

L'autre démarche se fonde sur le modèle de l'internationalisme libéral, qui affirme que le meilleur moyen de consolider la paix est d'instaurer rapidement la démocratie et l'économie de marché. Les critiques de ce modèle prétendent que le succès très limité de nombreux processus de consolidation de la paix provient justement d'une libéralisation politique et économique trop hâtive, les Etats concernés ne remplissant généralement pas les prémisses requises pour pareille mutation ${ }^{43}$.

\section{Tendances actuelles}

A part les deux conceptions mentionnées de la consolidation de la paix, le débat actuel est dominé en ce moment par deux questions, à savoir $1^{\circ} 1^{1}$ 'efficacité de la coopération au développement dans les Etats dits fragiles (aid effectiveness) et $2^{\circ}$ le succès des mesures de promotion de la paix pour la conclusion effective d'un traité de paix («macroprocessus» de paix).

\section{$\square$ Efficacité de la coopération au développement dans les Etats fragiles et les pays en conflit}

Depuis quelques années, les acteurs de la coopération au développement discutent de l'efficacité de celle-ci et concluent que la coopération au développement n'est efficace que quand les pays bénéficiaires disposent de politiques fiables et d'institutions compétentes ${ }^{44}$. Face à ce constat, on se demande comment la coopération au développement doit agir dans les Etats dits fragiles, qui sont souvent des pays en conflit. La Déclaration de Paris sur l'efficacité de la coopération au développement (Paris Declaration on Aid Effectiveness) reconnaît que ces pays exigent une attention particulière ${ }^{45}$. Or ils représentent presque la moitié de tous les pays bénéficiaires de la coopération au développement.

41 S.J. Stedman, D. Rothchild, E.M. Cousens, Ending Civil Wars: The Implementation of Peace Agreements, Boulder, Colorado, Lynne Rienner, 2002.

42 Voir la contribution de Xavier Tschumi Canosa au présent ouvrage.

43 R. Paris, At War's End: Building Peace after Civil Conflict, Cambridge, Cambridge University Press, 2004.

44 Paris High-Level Forum, Paris Declaration on Aid Effectiveness: Ownership, Harmonisation, Alignment, Results and Mutual Accountability, Washington, DC, The World Bank, 2005.

45 Ibid., p. 7. 
Malgré les difficultés rencontrées dans ces pays, les Etats donateurs ont décidé de continuer à s'engager en leur faveur et de maintenir leur aide, surtout parce qu'il s'agit de ne pas rendre leurs populations responsables de la faiblesse des prestations gouvernementales ${ }^{46}$.

\section{$\square$ Taux de réussite de la promotion de la paix - la question de l'évaluation}

Pour améliorer le taux de réussite des mesures de promotion de la paix, une des leçons du passé est qu'il est indispensable de professionnaliser beaucoup plus qu'autrefois la préparation et l'évaluation des interventions en faveur de la paix. Contrairement à la coopération au développement, c'est là un aveu nouveau pour la promotion de la paix. C'est seulement depuis que de nombreux donateurs ont déclaré ne plus vouloir soutenir que les initiatives dont l'efficacité pourrait être vérifiée que le débat sur l'évaluation de la promotion de la paix a démarré ${ }^{47}$.

Divers projets étudient les expériences faites en matière de promotion de la paix. Ainsi, la Joint Utstein Study ${ }^{48}$ a analysé les efforts de divers gouvernements en matière de promotion de la paix, tandis que l'étude Confronting War du projet «Reflecting on Peace» a analysé les leçons tirées par des ONG de leurs projets de promotion de la paix ${ }^{49}$. On dispose désormais d'une série de suggestions sur la manière d'évaluer les initiatives en faveur de la paix ${ }^{50}$.

46 Centre for the Future State, Signposts to More Effective States: Responding to Governance Challenges in Developing Countries, Brighton, Institute of Development Studies, 2005. T. Debiel, U. Terlinden, Promoting Good Governance in Post-Conflict Societies, Discussion Paper, Eschborn, Gesellschaft für Technische Zusammenarbeit, 2005. N. Leader, P. Colenso, Aid Instruments in Fragile States, PRDE Working Paper, n 5, January, London, Department for International Development, 2005. Organization for Economic Cooperation and Development (OECD), Principles for Good International Engagement in Fragile States: Learning and Advisory Process on Difficult partnerships (LAP), Paris, OECD, 2005.

47 C. Church, J. Shouldice, The Evaluation of Conflict Resolution Interventions, Part I and II, Londonderry, INCORE, 2002 and 2003. Journal of Peacebuilding and Development, vol. 2, $\mathrm{n}^{\circ}$ 1, 2005.

48 D. Smith, Towards a Strategic Framework for Peacebuilding: The Synthesis Report of the Joint Utstein Study on Peacebuilding, Oslo, Peace Research Institute Oslo, 2003.

49 M.B. Anderson, L. Olson, Confronting War: Critical Lessons for Peace Practitioners, Cambridge, MA, Reflecting on Peace Practice Project, Collaborative Development Action, 2003.

50 L. Fast, R. Neufeld, «Envisioning Success : Building Blocks for Strategic and Comprehensive Peacebuilding Impact Evaluation», Journal of Peacebuilding and Development, Evaluation Edition, vol. 2, $\mathrm{n}^{\mathrm{o}}$ 1, 2005. T. Paffenholz, The Evaluation of Peacebuilding Interventions, paper presented at the conference «Evaluation in der zivilen Konfliktbearbeitung», Evangelische Akademie Loccum, Loccum (Germany), 1-3 April 2005, published in German in Loccumer Protokolle, $\mathrm{n}^{\circ}$ 14, 2005, pp. 39 60. T. Paffenholz, L. Reychler, «Towards Better Policy and Programme Work in Conflict Zones: Introducing the "Aid for Peace" Approach», Journal of Peacebuilding and Development, vol. 2, n 2 , 2005, pp. 6-23. 
Pourquoi la coopération

au développement est-elle différente dans les pays en conflit?

Différentes raisons font que la coopération au développement se déroule autrement dans les pays en conflit.

\section{Risques sécuritaires}

Intervenir dans des territoires en conflit entraîne souvent des risques sécuritaires pour les collaborateurs et les infrastructures, de même que l'impossibilité d'accéder aux bénéficiaires ou aux partenaires d'un programme; le climat politique peut aussi entraver le travail dans les zones touchées ou le contact avec les parties au conflit. Les organisations de coopération au développement doivent donc être préparées à affronter ces gageures et tenir compte des risques potentiels dans leur planification.

\section{L'enfer est pavé de bonnes intentions}

Comme il a été vu plus haut, des projets de coopération au développement peuvent alimenter la dynamique conflictuelle malgré les meilleures intentions du monde. Plusieurs projets de développement au Sri Lanka et au Népal recrutaient par exemple des collaborateurs locaux sur la seule base de leurs qualifications professionnelles, sans tenir compte de la composition ethnique ou religieuse du personnel et de son appartenance à telle caste. Il en est résulté une préférence systématique pour certains groupes sociaux, ce qui a attisé le conflit existant.

\section{Paix et conflit sont des questions hautement politiques}

Dans les territoires en conflit, les acteurs de la coopération au développement affrontent généralement toute une série de problèmes politiques. Le premier partenaire des donateurs et des organisations d'entraide est normalement le gouvernement du pays. Mais dans les territoires en conflit, celui-ci est également partie au conflit, ce qui complique la coopération classique. Le problème s'aggrave si les organisations d'entraide doivent ou veulent collaborer avec des groupes armés non étatiques engagés dans des confrontations violentes avec le gouvernement, comme c'est le cas par exemple des LTTE au Sri Lanka. Ces groupes peuvent saboter les projets soutenus par le gouvernement ou menacer la sécurité personnelle des coopérants qui travaillent dans les régions sous le contrôle de ces groupes. Or ce sont souvent ces régions qui ont justement le plus besoin d'aide du fait qu'elles abritent une grande partie de la population nécessiteuse. S'il s'agit de poursuivre l'aide dans ces régions, il faut la coordonner plus efficacement. Malheureusement, la volonté de coopérer est souvent entravée par les intérêts politiques divergents des pays donateurs - leurs conceptions opposées de la «guerre contre le terrorisme», par exemple, ou leur préférence pour les solutions tantôt militaires, tantôt civiles. 
Pour planifier et évaluer les interventions dans les territoires en conflit, on peut recourir en principe aux instruments pratiqués depuis longtemps par la coopération au développement et l'aide humanitaire, ou à ceux fournis par la recherche. De nombreuses organisations de développement estiment cependant que leurs instruments de planification et d'évaluation sont applicables aussi bien dans les territoires en conflit qu'ailleurs et qu'il n'y a donc pas de raison de les adapter. En revanche, les organisations de promotion de la paix contestent le recours aux méthodes et instruments traditionnels de la coopération au développement. Les conflits armés seraient des phénomènes si complexes que les méthodes standard ne se prêteraient pas à la planification et à l'évaluation d'interventions en faveur de la paix.

A analyser de plus près les méthodes mondialement reconnues de planification et d'évaluation, on constate que ni l'un ni l'autre des raisonnements n'est entièrement faux ou juste. Si de nombreux critères standard sont parfaitement applicables aux territoires en conflit, d'autres doivent être adaptés à la situation ou élargis, parce que les situations de conflit sont effectivement plus complexes.

\section{Presque toutes les interventions de la coopération}

au développement peuvent aussi aider à promouvoir la paix

Même les activités de la coopération au développement qui ne sont pas axées directement sur la paix peuvent contribuer à promouvoir celle-ci, pour autant qu'on soit conscient de leur potentiel. Cela ne signifie pas cependant qu'il faille modifier désormais tous les plans d'intervention; la priorité d'un programme de développement reste sa contribution au développement du pays. Mais si le travail est inspiré par une démarche qui tienne compte des enjeux et des conflits potentiels, les acteurs examineront systématiquement s'il est possible de contribuer à promouvoir la paix tout en poursuivant les buts de la coopération au développement, et comment. Il est toujours plus nécessaire de concevoir ainsi la planification des projets, car sans paix, il n'y aura pas de développement social et économique durable.

Contributions de la coopération au développement et de l'aide humanitaire à la promotion de la paix

Il y a différents niveaux auxquels la coopération au développement et l'aide humanitaire peuvent contribuer à promouvoir la paix. Au niveau de la politique du développement («macropolitique»), elles peuvent concevoir des interventions ciblées; au niveau des politiques sectorielles, intégrer les paramètres conflit et paix dans les stratégies de développement des différents secteurs (santé, agriculture, eau, etc.); au niveau des projets, intégrer une démarche attentive aux paramètres paix et conflit dans les programmes et projets classiques d'entraide qui se déroulent dans le contexte d'un conflit armé et/ou soutenir directement des projets en faveur de la paix et des droits humains. Aujourd'hui déjà, les donateurs internationaux financent une vaste palette d'initiatives 
de ce genre. Nous nous pencherons maintenant sur chaque niveau et signalerons en conclusion les problèmes majeurs de chacun.

\section{Promotion de la paix au niveau de la politique du développement}

\section{$\square$ Approches et exemples}

Au niveau «macropolitique», à part la diplomatie, le state building, la politique des droits humains, la médiation et les bons offices ${ }^{51}$, les donateurs bi- et multilatéraux peuvent recourir à toute une série de stratégies: dialogue politique, étapes négociées (negotiated benchmarks), lignes rouges (bottom lines), conditionnalité des prestations d'entraide, sanctions. Des réseaux internationaux peuvent en outre être établis pour lutter contre les économies de guerre et influencer les parties au conflit ${ }^{52}$. Etudions brièvement ces stratégies et leurs implications pour les acteurs de la coopération au développement et de l'aide humanitaire.

Le dialogue politique implique un engagement à long terme, la plupart du temps avec pour interlocuteur un gouvernement disposé à coopérer. En envisageant des relations durables avec ce gouvernement, les donateurs espèrent pouvoir influencer sa politique de façon constructive. Un tel dialogue peut par exemple consister à sensibiliser le gouvernement au conflit, lequel est souvent ignoré ou minimisé pour des raisons politiques.

Dans la conditionnalité politique, on définit à quelles conditions l'aide sera accordée ou retirée. L'idée est de transformer positivement le conflit, par exemple en décourageant tel acteur essentiel de continuer à participer à la lutte armée ou de commettre de graves violations des droits humains. La reprise de l'aide est soumise à certaines conditions politiques. Exemples : à la suite de violations des droits humains commises par le gouvernement éthiopien à l'encontre d'opposants politiques, de grands donateurs comme l'Union européenne et la Banque mondiale cessèrent en décembre 2005 de financer le budget éthiopien; la même sanction fut appliquée au Népal après le «coup d'Etat royal»par lequel le roi avait dissous le Parlement et fait arrêter des chefs politiques, des activistes des droits humains et des journalistes.

Les étapes négociées sont des incitations positives qui peuvent être considérées comme la contrepartie de la conditionnalité. Si tel ou tel benchmark politique est atteint, les prestations d'entraide seront par exemple augmentées.

Les lignes rouges sont au contraire des normes minimales: l'engagement des donateurs cesse si elles sont franchies («si la situation ne s'améliore pas, nous mettrons fin à notre engagement»). Normalement, les conditions concrètes qui

51 T. Paffenholz, Konflikttransformation durch Vermittlung, op. cit. Idem, «Ansätze ziviler Konfliktbearbeitung», op. cit.

52 T. Paffenholz, «Peace and Conflict Sensitivity in International Cooperation: An Introductory Overview (2005)», International Politics and Society/Zeitschrift für Internationale Politik und Gesellschaft, $\mathrm{n}^{\circ}$ 4, 2005, pp. 63-82. P. Uvin, The Influence of Aid in Situations of Armed Conflict: A Synthesis and a Commentary on the Lessons Learned from Case Studies on the Limits and Scope for the Use of Development Assistance Incentives and Disincentives for Influencing Conflict Situations, paper for the Informal Talk Force on Conflict Peace and Development Cooperation, Paris, OECD, 1999. B. Wood, Development Dimensions of Conflict Prevention and Peace-Building, an independent study prepared for the Bureau of Crisis Prevention and Recovery, New York, United Nations Development Programme (UNDP), <http://www.undp.org/bcpr/ref/undp_pb_study3.pdf> 
doivent être améliorées sont spécifiées à l'avance - le comportement antidémocratique du roi du Népal, par exemple - et un délai est fixé pour y remédier.

Trouver des «points d'entrée » (entry points) pour les négociations de paix est une méthode classique de la médiation, qui est appliquée normalement par les acteurs diplomatiques ou les ONG. Mais il existe aussi des exemples heureux de «points d'entrée» élaborés en temps de guerre, en recourant cette fois à l'aide humanitaire (voir l'encadré sur le Mozambique).

\section{Soutien des négociations de paix au Salvador et au Mozambique à travers l'engagement humanitaire}

Pendant la guerre au Salvador, les représentants des organisations religieuses négocièrent par exemple de brefs cessez-le-feu pour vacciner les enfants. De même, le Comité international de la Croix-Rouge (CICR) parvint vers la fin de la guerre au Mozambique à établir des corridors humanitaires réservés à I'aide humanitaire et interdits aux belligérants. Ces deux interventions contribuèrent notablement à accélérer le processus de paix. Après qu'on eut constaté au Salvador que les parties au conflit étaient capables de négocier des cessez-le-feu, la pression augmenta afin qu'elles entament des négociations de paix. Au Mozambique, de plus en plus de personnes purent quitter les zones de combat par les corridors humanitaires, ce qui mit sous pression l'une des parties au conflit et renforça sa disposition à s'asseoir à la table des négociations.

Les réseaux internationaux de lutte contre les économies de guerre essaient d'assécher les finances des belligérants en contrôlant leurs canaux commerciaux. C'est ce que fait avec un certain succès le processus dit de Kimberley, qui tente de juguler le commerce des «diamants du sang». Dans d'autres processus analogues, encore en cours (transparence des revenus tirés des gisements pétroliers des pays en conflit; alternatives au trafic de la drogue en Afghanistan ou en Colombie), le succès se fait toujours attendre.

\section{$\square$ Leçons et défis}

Les interventions de la coopération au développement et de l'aide humanitaire dans les pays qui connaissent des tensions politiques et des conflits armés ont d'abord renforcé l'aspect politique du développement, ce qui a eu pour effet de rendre encore plus nécessaire la collaboration des politiciens, des diplomates et des coopérants dans les pays donateurs. Cela n'a pas été toujours simple à réaliser, au vu des différents intérêts en jeu, mais il est manifeste que de plus en plus de pays élaborent des stratégies nationales cohérentes, à l'instar du Global Conflict Prevention Pool (GCPP) de Grande-Bretagne, créé en 2001, et qui représente une nouvelle manière d'aborder la prévention des conflits. En regroupant les ressources des ministères de la défense et de la politique étrangère, du Commonwealth Office et du Department for International Development (DFID), instance responsable de la coopération au développement, le GCPP peut concevoir une démarche stratégique cohérente. Il a mis ainsi au point une vision intégrée $\mathrm{du}$ traitement des conflits, et pour plusieurs régions (Balkans, Afghanistan, Proche-Orient et Afrique du nord, Népal et Indonésie), des synergies ont pu être réalisées en matière de prévention des conflits. En Suisse, on élabore également des stratégies communes pour différents pays, comme le Népal ${ }^{53}$.

53 Voir la contribution de Günther Baechler et Jörg Frieden au présent ouvrage. 
La nécessité pour la coopération au développement de se montrer plus «politique» a accru la demande en collaborateurs qualifiés. Il y a là un vrai problème, du fait que les carrières des diplomates et des coopérants continuent généralement à se dérouler séparément.

Une autre leçon importante est que la plupart des interventions politiques partent de l'idée que l'aide au développement et la reconnaissance internationale des parties au conflit seraient importantes. Or ces interventions ne peuvent généralement exercer une influence sur la situation d'un pays en conflit que si celui-ci dépend dans une large mesure de ses donateurs et ne veut perdre ni leur reconnaissance ni leurs ressources. Un pays exportateur de pétrole comme l'Angola, en revanche, ne dépend pas de l'aide au développement et est donc plus ou moins immunisé contre les pressions des donateurs.

Les mesures politiques peuvent malgré tout être très efficaces, surtout si elles sont concertées internationalement. Il se pose toutefois une série de problèmes. Les donateurs détestent prendre des décisions politiques dures, parce qu'ils ne veulent pas aggraver leurs relations diplomatiques avec tel gouvernement partenaire, même si celui-ci est devenu partie au conflit. Il apparaît ici des incompatibilités notables entre la promotion de la paix, la coopération au développement et les intérêts diplomatiques.

Depuis la «guerre contre le terrorisme» qui polarise les relations internationales, ces dilemmes ont encore augmenté. La plupart des groupes armés en lutte contre leur gouvernement sont perçus désormais comme des terroristes, ce qui rend encore plus difficiles la cohérence des réactions internationales et les solutions négociées par le dialogue. A cause de la «guerre contre le terrorisme», de nombreux gouvernements reçoivent de nouveau de l'aide militaire et favorisent donc de nouveau les solutions militaires à leurs conflits armés. De ce fait, la «bonne gouvernance» a également perdu de sa pertinence dans la coopération au développement. D'une certaine façon, le niveau du raisonnement est retombé à celui de la guerre froide, où l'on soutenait les gouvernements autoritaires corrompus qui se trouvaient dans le bon camp.

Il n'est pas facile de surmonter ces difficultés. Il faut continuer à sensibiliser les esprits aux problèmes des conflits, surtout auprès des donateurs.

\section{Promotion de la paix à travers une politique sectorielle attentive aux conflits}

Dans les pays en développement, la coopération au développement peut également contribuer à promouvoir la paix en intégrant les paramètres paix et conflit dans ses politiques sectorielles. La plupart du temps, on commencera par analyser à fond les sources de conflit et à élucider leur rapport avec les secteurs respectifs de la coopération au développement.

Les expériences montrent que le défi majeur est l'analyse des politiques sectorielles de la coopération au développement dans la perspective des conflits. Si ce potentiel a été trop peu exploité jusqu'ici, c'est que la plupart des donateurs et des agences se concentrent sur le niveau des projets quand il s'agit de réaliser une coopération au développement attentive aux conflits. 


\section{Politique sectorielle attentive aux conflits au Yémen et en Ouganda}

Les causes profondes du conflit yéménite sont la pénurie des ressources naturelles (à commencer par l'eau). Le secteur hydrique joue donc un rôle décisif dans la guerre et la paix. On pourra alors promouvoir la paix en donnant accès à l'eau à la population, tout en veillant à une répartition équitable. Les acteurs du développement - gouvernement, donateurs, organisations locales ou internationales d'entraide - doivent prendre d'abord conscience de ces interdépendances et agir ensuite de façon cohérente.

Les causes du conflit ougandais sont nombreuses, mais l'une d'elles est la discrimination dont souffrent les populations du nord sur le plan de la participation politique et du développement général. A moyen et à long terme, on pourra contribuer à promouvoir la paix en impliquant systématiquement la partie nord du pays dans la planification des différents secteurs.

Une coopération au développement attentive à la paix et aux conflits au niveau des projets

Les spécialistes du développement admettent généralement qu'au niveau des projets, les acteurs disposent de trois options pour travailler dans les régions en conflit ${ }^{54}$ :

$\square$ travailler en dehors du conflit (working around conflict): le conflit est considéré comme un facteur de risque négatif, qu'il s'agit d'éviter;

$\checkmark$ travailler dans le cadre du conflit (working in conflict): les acteurs sont conscients (jusque dans une certaine mesure) de ce que la coopération au développement peut affecter le conflit; ils s'efforcent d'éviter les effets néfastes de la coopération au développement sur le conflit (do no harm);

$\checkmark$ travailler sur le conflit (working on conflict): les acteurs savent que la coopération au développement peut aussi contribuer à promouvoir la paix.

Les coopérants et les acteurs humanitaires peuvent donc aussi bien recourir à des méthodes attentives à la paix et au conflit dans leurs projets traditionnels que financer ou réaliser directement des projets en faveur de la paix. La première option sera traitée ici, la seconde dans la section suivante («Comment fonctionne la promotion directe de la paix au niveau des projets?»).

\section{$\square$ En quoi consistent les méthodes attentives à la paix et aux conflits} dans les projets de coopération au développement et d'aide humanitaire?

Les programmes humanitaires et d'aide au développement ont en général d'autres buts que de promouvoir la paix; leur objectif premier est de contribuer au développement économique et social d'un pays ou d'une région, ou d'atténuer la souffrance humaine. Prendre en compte les paramètres paix et conflit ne s'impose que lorsque les projets correspondants sont confrontés à des conflits armés latents ou manifestes. A ce stade, il existe différentes approches, mais leur but sera toujours de concevoir les projets de façon à ce que les risques liés au conflit soient minimisés, que les projets n'aggravent pas la dynamique conflictuelle et qu'on ait déterminé s'ils contribuent à promouvoir la paix, ne serait-ce qu'indirectement.

54 J. Goodhand, Armed Conflict, Poverty and Chronic Poverty, Working Paper, $\mathrm{n}^{\mathrm{o}}$ 6, Conflict Prevention and Resolution Center (CPRC), University of Manchester, 2001. 


\section{Bonnes pratiques au Burundi}

L'Organisation d'appui à l'autopromotion (OAP) est une ONG locale (soutenue par I'Institut universitaire d'études du développement - IUED) qui travaille dans la province burundaise de Bujumbura. Depuis douze ans, I'OAP aide les communautés d'une dizaine de communes à développer des activités lucratives, à accéder à des facilités de crédit et à réaliser des projets de construction et d'assainissement des infrastructures et des habitations. L'OAP effectue son travail dans un contexte de forte tension et de confrontations armées fréquentes. Elle vise à améliorer la situation socio-économique, ce qui contribue à apaiser le climat général. L'organisation suit les communautés non seulement en période de calme, mais aussi en cas de troubles. Ses activités sont manifestement très appréciées dans la province de Bujumbura. Sur le plan économique, elle a obtenu des résultats concrets avec la construction et l'assainissement des infrastructures ainsi qu'avec des programmes de formation, qui ont nettement amélioré la situation socio-économique et fait reculer la pauvreté. L'engagement de l'OAP a aussi des effets remarquables sur la confiance en soi et la fierté de la population rurale, dont la mentalité a changé. Ce phénomène se remarque non seulement quand on parle avec les instances officielles, installées en ville, mais surtout si l'on observe directement le comportement de la population à divers moments et en des endroits différents, ou que l'on discute avec les associations locales. On découvre alors des exemples encourageants.

$\square$ Esprit d'initiative pour résoudre des problèmes personnels. Des douzaines de personnes (membres ou non de I'organisation) s'annoncent chaque lundi au siège de I'OAP pour exposer leurs problèmes, demander conseil, présenter des projets, etc. L'introduction de la "journée portes ouvertes» du lundi s'avère très efficace. Les gens affluent parce qu'ils ont confiance dans I'OAP.

- Autodétermination. Avec le soutien et les conseils de l'OAP (rôle de médiateur), les gens osent s'opposer aux mesures arbitraires et aux injustices commises par certaines instances, par exemple en insistant sur leur droit au sol.

- Conscientisation. Parmi les acteurs locaux encadrés par I'OAP, on trouve toujours plus de personnes qui utilisent les systèmes bancaires modernes, ont l'esprit ouvert au dialogue interethnique, manifestent de l'intérêt pour les nouveautés et prennent au sérieux leur rôle et leur responsabilité dans les comités administratifs.

ـ Dépôt de nouvelles requêtes pour la fondation d'associations et le suivi de leurs activités par l'OAP.

L'exemple de l'OAP montre que le développement socio-économique de la base peut influencer positivement l'attitude foncière des gens quand la population prend elle-même l'initiative. C'est là évidemment un facteur très important pour instaurer une paix durable.

Daniel Fino, IUED

On distingue deux types d'approches pour développer la sensibilité à la paix et aux conflits. Celles du premier type intègrent les analyses classiques du conflit dans les projets ${ }^{55}$. Dans la pratique, une telle analyse sera effectuée soit avant le début, soit au cours d'un projet, qui sera alors adapté en conséquence. L'utilité majeure de cette approche a été jusqu'ici de sensibiliser les esprits; les acteurs du développement commencent désormais à saisir l'incidence d'un conflit sur leurs projets. Sa plus grande faiblesse est le manque de lien entre analyse et mise en œuvre. L'analyse fournit souvent des résultats très utiles, mais qui n'entrent qu'insuffisamment dans l'élaboration du projet. En outre, on néglige

55 Voir les nombreux exemples énumérés dans Resource Pack (Africa Peace Forum, Center for Conflict Resolution, Consortium of Humanitarian Agencies, Forum on Early Warning and Early Response, International Alert and Saferworld), Conflict-sensitive Approaches to Development, Humanitarian Assistance and Peacebuilding: A Resource Pack, 2004, accessible sur <http://www.conflictsensitivity. org $>$. 
souvent d'associer les stakeholders du projet à la planification et à l'évaluation. Il y a donc un risque que l'analyse reste un acte isolé au lieu d'être intégrée dans la planification normale.

Les approches du second type tentent en revanche d'associer systématiquement l'analyse des conflits avec la mise en œuvre pratique. On peut distinguer ici trois approches :

- celle de Mary B. Anderson, Local Capacities for Peace, plus connue sous le nom de do no harm ${ }^{56}$;

๖ celle de Kenneth Bush, Hands-on PCIA ${ }^{57}$;

๑ celle de Thania Paffenholz et Luc Reychler, Aid for Peace ${ }^{58}$.

\section{Approches différentes \\ en matière de coopération au développement attentive à la paix et aux conflits}

L'approche do no harm se fonde sur les leçons du projet Local Capacities for Peace, qui propose une grille d'analyse pour la planification de projets et des listes de vérification pour évaluer les effets positifs et négatifs d'un projet. On peut ainsi vérifier la conception d'un projet et, sur la base des résultats, l'adapter de façon à ce que les capacités locales de promotion de la paix soient renforcées le plus possible. L'approche do no harm est pratiquée essentiellement dans les territoires en crise par les ONG internationales, qui y forment leurs partenaires locaux. Do no harm est donc une grille d'évaluation autonome, qui complète la planification et le monitoring normal. Son application large et la conscientisation qu'elle opère sur place peuvent être considérées comme des succès.

Hands-on PCIA est une approche qui guide les participants d'un atelier (généralement les collaborateurs d'un projet) à travers diverses étapes d'analyse et d'évaluation (analyse des conflits, calcul des risques, appréciation des chances). Elle permettra d'adapter l'intervention en conséquence. Le PCIA élargi de Bush a été publié dans quelques langues indigènes pour permettre aux acteurs locaux de le pratiquer de façon autonome.

L'approche Aid for Peace est une assistance à la planification et à l'évaluation d'interventions de promotion de la paix, de la coopération au développement et des acteurs humanitaires dans des territoires en conflit. II s'agit d'un cadre méthodologique commun destiné à des acteurs et des niveaux d'intervention différents (niveau général, niveau sectoriel, niveau des projets). Le cadre Aid for Peace se compose de quatre parties: $1^{\circ}$ spécification des besoins en matière de promotion de la paix dans un pays, territoire ou région donné; $2^{0}$ évaluation de l'incidence sur la paix et le conflit de l'intervention projetée ou réalisée; $3^{0}$ évaluation des effets attendus ou déjà survenus du conflit sur l'intervention/le projet (risques liés au conflit); $4^{0}$ analyse des effets attendus ou déjà survenus de l'intervention sur la dynamique conflictuelle et le processus de paix.

56 M. B. Anderson, «Experiences with Impact Assessment: Can We Know What Good We Do?», in Berghof Handbook for Conflict Transformation, Berlin, Berghof Research Center for Constructive Conflict Management, 2004, <http://www.berghof-handbook.net>.

57 PCIA: Peace and Conflict Impact Assessment. K. Bush, «Field Notes: Fighting Commodities and Disempowerment in the Development Industry. Things I learned from PCIA in Habarana and Mindanao », in New Trends in PCIA, Dialogue Series, n 4, 2005, Berghof Handbook for Conflict Transformation, op. cit.

58 T. Paffenholz, «Third-Generation PCIA: Introducing the Aid for Peace Approach», in New Trends in PCIA, Dialogue Series, $\mathrm{n}^{\circ}$ 4, 2005, Berghof Handbook for Conflict Transformation, op. cit. T. Paffenholz, L. Reychler, «Towards Better Policy and Programme Work in Conflict Zones», op. cit. 


\section{$\square$ Leçons et défis}

Les facteurs paix et conflit sont bien entrés dans l'agenda courant des donateurs et des organisations internationales d'entraide. "Paix et conflit» fait désormais partie des thèmes «transversaux» principaux, au même titre que le genre (gender), l'environnement, la gouvernance ou le sida. La prévention des conflits et la promotion de la paix sont aussi des thèmes prioritaires de la Direction du développement et de la coopération (DDC). Toutes les organisations d'entraide et les donateurs d'une certaine taille ont créé des unités spécialisées ou des postes de conseillers en matière de conflit.

Intégrer le sujet dans le courant normal (mainstreaming) de la coopération au développement soulève cependant toute une série de problèmes. L'un d'eux est la structure de nombre d'organisations donatrices: alors que les unités dites géographiques gèrent les programmes, donc les fonds, celles dites thématiques étudient les thèmes transversaux et conseillent les unités géographiques. Cette structure cache à son tour deux problèmes: $1^{\circ}$ en général, les unités géographiques ne sont pas tenues de se procurer des conseils; $2^{\circ}$ il existe aussi une concurrence entre les unités thématiques, qui sont peu coordonnées entre elles. Le grand nombre de thèmes et d'unités transversales fait que les collaborateurs se sentent incapables d'intégrer tous les thèmes dans leur travail habituel. Il en résulte qu'ils développent souvent des résistances et n'assimilent qu'à contrecœur un nouveau thème.

\section{Méthodes de mainstreaming novatrices}

Face à ces problèmes de gestion, la DDC a introduit le Conflict Sensitive Programme Management (CSPM). La manière dont ce système a été appliqué dans le cas du programme de la DDC pour le Népal peut être considérée comme exemplaire ${ }^{\mathrm{a}}$.

Pour gérer le problème du grand nombre de thèmes transversaux, l'agence suédoise de coopération internationale pour le développement (SIDA) a commencé par réduire ce nombre à quelques-uns et par créer un réseau des différentes unités thématiques. Ce réseau organise des séances de formation et de stratégie communes, avec pour but de trouver des solutions collectives qui correspondent au cadre global de sida pour la lutte contre la pauvreté.

Pour pallier l'absence de communication entre ses divisions spécialisées et ses unités géographiques, I'Agence allemande de coopération technique (GTZ) a créé dans ces dernières des guides thématiques dits stratégiques, qui coordonnent la prise en compte de certains thèmes transversaux et de leur contexte régional dans les opérations de routine (mainstreaming). Les unités géographiques se voient attribuer du personnel supplémentaire pour cette tâche. Des unités spéciales fournissent instruction et appui. Le ministère allemand de la coopération économique et du développement (BMz) a également institué une procédure de routine obligatoire pour intégrer la prise en compte de la paix et des conflits dans la coopération au développement et a adopté l'approche Aid for Peace comme méthode d'évaluation de la paix et des conflits (peace and conflict assessment). Toutes les agences doivent désormais adapter leurs procédures de planification, de mise en œuvre et d'évaluation pour garantir que les paramètres paix et conflit soient pris en compte lors d'activités dans un pays en conflit. La qualification de "pays en conflit» est décidée une fois par an par le BMZ, sur la base d'une série d'indicateurs d'alerte analysés par le Deutsches Übersee-Institut de Hambourg (depuis janvier 2006 GIGA, soit German Institute of Global and Area Studies - Leibniz Institut für Globale und Regionale Studien). Les résultats de ces analyses sont considérés comme informations internes et ne sont pas publiés.

a Voir la contribution de Günther Baechler et Jörg Frieden au présent ouvrage. 
Un autre problème est la mise en œuvre locale dans les programmes de coopération au développement et d'aide humanitaire. Aménager les programmes en tenant compte des conflits impose des exigences considérables aux responsables des programmes et des projets. Il leur faut par exemple faire preuve de souplesse lors de la budgétisation et de la mise en œuvre des projets. Pour assurer la sécurité du personnel, il faut avoir le sens des responsabilités et savoir gérer les risques. Le niveau des projets doit être coordonné avec le niveau politique. Il faut recourir à de nouveaux instruments ou en appliquer différemment d'anciens. Il faut enfin bien comprendre l'ensemble du problème et disposer du temps nécessaire et du personnel convenable. Or ces aspects de la gestion sont souvent négligés.

\section{Comment fonctionne la promotion directe de la paix au niveau des projets?}

Les donateurs et les organisations d'entraide financent ou réalisent un grand nombre de projets en faveur de la paix. Tel qu'il est utilisé aujourd'hui, le terme de «promotion de la paix» recouvre en effet des activités très diverses: désarmement, démobilisation et réinsertion sociale d'anciens combattants, formation à la résolution des conflits ou aux négociations, éducation pour la paix, assistance aux groupes pacifistes de la société civile ou aux activités qui visent à restaurer des relations humaines détruites (projets de réconciliation ou de dialogue, encouragement des médias ou enseignement de l'objectivité journalistique, etc.). Des mesures telles que le soutien aux tribunaux jugeant les criminels de guerre ou aux commissions de réconciliation, de même que la promotion de structures fédéralistes, font aussi partie de la promotion de la paix.

Parmi ces activités, un grand nombre s'inspirent de l'école «transformiste», en particulier de l'approche middle-out de Lederach ${ }^{59}$, car ce sont la plupart du temps les acteurs et institutions du niveau médian de la société (couche 2) qui bénéficient de soutien. La manière dont ce système théorique a été mis en pratique a entraîné une série de résultats positifs, mais a soulevé aussi quelques problèmes.

\section{$\square$ Prestations positives}

Il y a unanimité pour dire que dans les pays en conflit, c'est aux acteurs nationaux d'assumer le rôle principal en matière de promotion de la paix. Le rôle des acteurs externes devrait se limiter à soutenir les acteurs nationaux ${ }^{60}$. On reconnaît également que pour reconstruire la paix, les initiatives indépendantes des gouvernements sont tout aussi nécessaires que les efforts diplomatiques officiels et officieux.

Sur le plan international, certains thèmes pacifistes comme les armes légères ou l'économie de guerre ont pu être inscrits à l'agenda. De même, un lobbysme fécond a attiré l'attention du monde sur certains processus de paix dans des pays en conflit, à quoi a aussi contribué l'émergence d'une société civile mondiale ${ }^{61}$.

\footnotetext{
59 Voir plus haut la section «Augmentation fulgurante des activités de la promotion de la paix à partir du milieu des années $1990 »$.

60 J.P. Lederach, Building Peace: Sustainable Reconciliation in Divided Societies, op. cit.

61 M. Kaldor, Global Civil Society: An answer to War, Cambridge, UK, Polity Press, 2003.
} 
Le processus de Kimberley pour interdire le commerce des «diamants de la guerre» remonte par exemple à une campagne de la société civile mondiale. Il a pu être repris ensuite dans une initiative gouvernementale internationale dans laquelle la Suisse joue un rôle actif.

Un autre succès à relever est qu'en matière de promotion de la paix. la collaboration entre représentants du gouvernement et ONG internationales est devenue affaire de routine dans plusieurs pays, chacun étant disposé à apprendre de l'autre.

\section{Centre pour la promotion de la paix (КоғF) à Berne, <www.swisspeace.org >}

Installé chez Swisspeace (Fondation suisse pour la paix), le Centre pour la promotion de la paix a été fondé en 2001 par le Département fédéral des affaires étrangères (DFAE) et de nombreuses ONG suisses. II met à la disposition de tous les acteurs suisses - que ce soit des agences gouvernementales ou des organisations non gouvernementales - des analyses, des conseils et des cours de formation, et les aide à se mettre en réseau.

Dans les pays en conflit, la collaboration entre ONG locales, nationales et internationales a en outre aidé considérablement à faire entendre la voix des populations en Europe ou aux Etats-Unis.

\section{$\square$ Effets négatifs concomitants}

Le soutien que des donateurs externes apportent aux ONG va la plupart du temps à des ONG internationales, qui le redistribuent à des ONG nationales ou communales dirigées par des membres de l'élite. Si certaines de ces dernières prétendent être en liaison avec des groupes et communautés locaux, la plupart n'ont qu'un faible nombre d'affiliés. Il est rare qu'elles jouissent d'une représentativité nationale, équilibrée du point de vue politique ou ethnique, et souvent elles sont fortement liées à l'establishment politique par des liens de parenté. Face à leur multiplication fulgurante dans les pays en conflit, quelque auteurs parlent

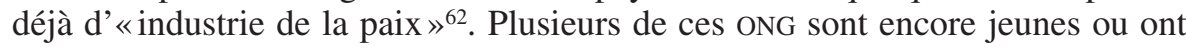
transféré leurs priorités du développement à la promotion de la paix, ce qui a souvent abouti à affaiblir les communautés locales originales ${ }^{63}$.

De nouvelles études sur l'efficacité du travail en faveur de la paix au Sri Lanka confirment ce constat. Les pays donateurs et les ONG ont toujours supposé jusqu'ici que les initiatives lancées par les ONG nationales auraient automatiquement une incidence sur le conflit au plus haut niveau. Or, ces nouvelles études démontrent que le niveau supérieur du processus de paix ne peut être atteint automatiquement que si certaines conditions sont remplies. L'étude «Reflecting on Peace Practice Project» (RPP) montre que pour promouvoir efficacement la paix, il faut soutenir à fond soit certaines personnes clés (key persons), soit une

62 B. Moltmann, «Die "Friedensindustrie" als Konfliktmotor? Das Beispiel Nordirland», in Kriege als (Über)Lebenswelten. Schattenglobalisierung, Kriegsökonomien und Inseln der Zivilität, S. Kurtenbach, P. Lock, Bonn, Dietz Verlag, 2004, pp. 236-248.

63 B. Pouligny, «Civil Society and Post-Conflict Peacebuilding: Ambiguities of International Programmes Aimed at Building "New" Societies», Security Dialogue, vol. 36, n 4, 2005, pp. 495-510. K. Bush, «Alice through the Looking Glass», in New Trends in PCIA, Dialogue Series, $\mathrm{n}^{\circ}$ 4, 2005 , Berghof Handbook for Conflict Transformation, op. cit. 
masse critique d'un très grand nombre de gens ${ }^{64}$. Malgré cela, des ONG qui, faute d'une base massive, n'atteignent qu'un nombre limité de gens continuent à toucher la majeure partie des fonds. C'est qu'il est évidemment plus simple pour les ONG internationales de collaborer avec les ONG de l'élite urbaine, qui parlent le langage des donateurs et comprennent la logique des projets proposés, que d'entrer en rapport avec les représentants des communautés locales.

\section{«Commercialisation » du travail en faveur de la paix au Sri Lanka}

Au Sri Lanka, les causes profondes du conflit sont la combinaison du sous-développement et de la discrimination, d'une part, avec la mobilisation ethnique, politique et religieuse, de l'autre. La concentration dans le sud du pays du pouvoir politique et économique entrave systématiquement le développement du nord et de l'est, pauvres en matières premières et peuplés majoritairement de Tamouls. Avec le temps, langue, appartenance ethnique et religion ont attisé le conflit. En 1956, pour s'affranchir de la prédominance persistante de l'élite anglophone occidentalisée, le gouvernement déclara le cinghalais langue nationale. Le pouvoir passa aux mains de l'élite cinghalaise, en majorité bouddhiste. II devint difficile pour les Tamouls bien formés d'accéder aux postes convoités du gouvernement. Une politique axée sur les Cinghalais aboutit à discriminer la population d'expression tamoule, qui avait été privilégiée dans l'administration à l'époque coloniale, et contribua largement à l'expansion du conflit et à la formation d'une résistance tamoule.

Le Sri Lanka a connu de nombreuses formes d'engagement social et politique de la part de groupes locaux et nationaux, mais avec la professionnalisation et la commercialisation du travail en faveur de la paix, ce dernier a été monopolisé par quelques ONG d'élite, installées la plupart du temps dans la capitale, à Colombo. L'engagement social «normal» de la population a diminué et le travail local en faveur de la paix a perdu en importance au fur et à mesure que les ONG nationales des deux parties au conflit perdaient leur lien avec la population et les communautés villageoises. Or, pour résoudre le conflit ethnique qui divise le pays, il serait indispensable de mobiliser les gens en faveur de la paix, ce dont les ONG nationales sont justement incapables. L'influence de la population civile en matière de promotion de la paix au Sri Lanka est donc restée très limitée ${ }^{a}$.

a C. Orjuela, Civil Society in Civil War: Peace Work and Identity Politics in Sri Lanka, PhD Dissertation, Department of Peace and Development Research, Göteborg University, 2004.

Une autre chose qui a changé est le rapport entre la recherche d'une part et les donateurs et organisations d'entraide d'autre part. La communauté des chercheurs scientifiques est de moins en moins consultée par les donateurs internationaux et les organisations d'entraide. Désormais, c'est un petit cercle de grandes ONG internationales très en vue, issues la plupart du monde anglophone, qui monopolise le discours pratique. Il faut donc distinguer nettement entre la théorie scientifique de la promotion de la paix et le discours des ONG internationales sur le même sujet. Donateurs et ONG internationales sont étroitement imbriqués. Les ONG internationales répondent au besoin des donateurs de voir des résultats rapides sur les dossiers d'actualité; en contrepartie, les donateurs assurent le financement de base et allouent des fonds aux projets des ONG. Les praticiens établis dans les capitales et sur place se voient fournir des réponses rapides aux problèmes conceptionnels, réponses auxquelles il manque cependant des preuves empiriques fiables, la réflexion universitaire critique et la variété des points de vue. 


\section{Evaluation du programme de paix "People to People» (P2P) dans le conflit israélo-palestinien}

Le conflit israélo-palestinien a des racines profondes et une longue histoire, mais on sait qu'il est actuellement au point mort. Malgré des décennies de luttes et de palabres, les fronts n'ont guère évolué. Résoudre un conflit aussi long, aux conséquences sociales aussi radicales, exige une réconciliation générale et l'acceptation par les parties du principe de la coexistence pacifique, ce qui ne pourra résulter que d'un profond changement des mentalités, des deux côtés. Cela dit, les accords d'Oslo (1993 et 1995) entre Israël et l'Organisation de libération de la Palestine (oLP) avaient quand même défini le cadre d'une foule de projets regroupés dans le programme "People to people» (P2P), qui devait encourager Israéliens et Palestiniens à mieux se comprendre. Ce programme a financé 165 projets mixtes (ateliers de collaboration professionnelle, groupes de loisirs, festivals de cinéma, activités écologiques, diffusion de livres, rencontres de journalistes, partenariats scolaires) pour promouvoir le dialogue israélo-palestinien et les relations mutuelles. II doit pourtant être qualifié d'échec, car s'il a influencé la perception individuelle et les relations des participants entre eux, les questions de l'opinion personnelle sur l'autre nation (et non seulement sur tel individu) n'ont la plupart du temps pas été abordées. Ces rencontres n'ont donc pas joué de rôle important dans le processus de paix, bien que cela eût été urgent en période de violence extrême ${ }^{\mathrm{a}}$.

a R. Taha, Grassroots Participation in Confidence Building: P2P Evaluation of the Past and Recommendations for the Future, draft, February 2003, Geneva, <http://www.unidir.org>. A. Atieh et al., Peace in the Middle East: P2P and the IsraeliPalestinian Conflict, Geneva, United Nations, 2004.

\section{Conclusions et problèmes futurs}

Le présent article a tenté de présenter dans les grandes lignes les rapports entre conflits armés et promotion de la paix, d'une part, et coopération au développement et aide humanitaire, de l'autre. Nous avons vu que la promotion de la paix n'est pas un thème nouveau puisqu'elle a joué un rôle dès l'Antiquité. Considérer la coopération au développement sous l'angle guerre et paix est cependant une conquête beaucoup plus récente, qui remonte en fait à 1994, après la tragédie rwandaise. Aujourd'hui, guerre et paix sont parmi les thèmes transversaux les plus importants de l'agenda pour le développement; les acteurs de la coopération au développement financent une foule de projets en faveur de la paix ou sont responsables de leur réalisation.

Après le génocide rwandais, le thème guerre et paix fut d'abord traité de façon très politique, avant qu'on se mette à aborder de plus près ses aspects «instrumentaux ». Ces dernières années, on a mis au point toute une série de «boîtes à outils» (toolboxes), mais peu d'entre elles offrent une approche complète qui en ferait des mécanismes utilisables par des acteurs différents à tous les niveaux d'intervention. La condition primordiale d'une bonne approche est le lien systématique entre l'analyse du champ conflictuel et la mise en œuvre progressive des interventions correspondantes, ou encore le lien entre la transformation du conflit et les exigences professionnelles et opérationnelles posées aux concepteurs et réalisateurs de programmes et projets.

En réalité, la promotion de la paix ne fait que commencer à donner des résultats pratiques concrets sur le terrain. Il n'existe jusqu'ici qu'un petit nombre de bons projets pilotes, mais une grande quantité d'exposés théoriques et de nombreux efforts pour inscrire ces préoccupations dans les opérations de routine des centrales. Il reste plusieurs problèmes, dont certains sont esquissés ici. 
Pour maîtriser les problèmes du travail dans les territoires en conflit, il convient de reprendre le débat sur la paix et les conflits à un niveau plus politique ${ }^{65}$. C'est que les modèles politiques existants ne sont souvent pas mis en œuvre dans leur totalité, les donateurs ayant de la peine à conduire une politique cohérente dans des pays fragiles, minés par des conflits. Or paix et conflit sont des questions éminemment politiques: tel gouvernement partenaire devient partie au conflit; la nécessité de parler avec des acteurs non étatiques armés est incontournable puisque ceux-ci contrôlent souvent de grandes parties du pays auxquelles il faut pouvoir accéder pour soutenir la population. Il en ressort que la collaboration entre diplomates et acteurs du développement doit s'améliorer considérablement.

De la conception instrumentale de l'attention à la paix et aux conflits à une vue plus générale

Comme pour beaucoup d'autres thèmes transversaux de la coopération au développement, celui du conflit a été abordé par plusieurs organisations d'entraide sous la forme d'une stratégie réduite à des «outils» (tools) au niveau des projets. Or il est nécessaire de l'aborder dans une perspective intégrée, c'est-à-dire de combiner les aspects politiques, opérationnels et gestionnels.

\section{$\square$ Consolider le renforcement des capacités dans le Sud}

Il existe toute une série de programmes de formation, en particulier pour le do no harm, mais il faut en faire plus pour renforcer le renforcement des capacités (capacity-building), surtout dans le Sud. Il faut des partenariats avec des institutions du Sud pour exploiter le savoir local dans la coopération internationale en tenant compte des conflits. Il faut éviter d'abandonner le champ de la promotion de la paix aux seules organisations d'entraide occidentales et à leurs conseillers. Cela dit, il convient aussi de continuer à sensibiliser le Nord aux rapports entre conflit et coopération au développement.

\section{$\square$ Fortifier la société civile des pays en conflit plutôt que soutenir les ONG}

Sous l'étiquette «soutien à la société civile», la promotion de la paix a connu elle aussi autrefois un soutien très marqué aux ONG nationales et internationales, comme si la société civile ne se composait que d'ONG. Les constats empiriques prouvent que ce soutien aux ONG de l'élite urbaine (la plupart du temps) doit être considéré d'un œil plus critique. Souvent, des groupes sociaux et des groupements pacifistes locaux ont été affaiblis alors qu'ils seraient de première importance pour l'édification d'une paix durable.

65 K. Bush, «Field Notes : Fighting Commodities and Disempowerment in the Development Industry », op. cit. Idem, «Alice through the Looking Glass», op. cit. T. Paffenholz, «Third-Generation PCIA: Introducing the Aid for Peace Approach», op. cit. Idem, «More Field Notes», in New Trends in PCIA, Dialogue Series, n 4, 2005, Berghof Handbook for Conflict Transformation, op. cit. 